\title{
Fundamental stellar parameters of three Kepler stars accurately constrained by lithium abundance and rotation
}

\author{
K. Liu, S. L. Bi, T. D. Li, Z. J. Tian, and Z. S. Ge \\ Department of Astronomy, Beijing Normal University, 100875 Beijing, PR China \\ e-mail: liukang@mail.bnu.edu.cn; bisl@bnu.edu.cn \\ Received 18 December 2013 / Accepted 27 January 2014

\section{ABSTRACT}

\begin{abstract}
Aims. The lithium abundances of KIC 11395018 and KIC 10920273 are not compatible with the age of these stars, which is deduced by asteroseismology. To explain this phenomenon, we investigated the possible evolutionary status and performed a seismological analysis of the three stars KIC 11395018, KIC 10273246, and KIC 10920273.

Methods. Using the Yale Rotating Stellar Evolution Code (YREC), we constructed stellar models that include diffusion and extramixing caused by rotation. In addition to the general observed properties, we considered two constraints, the lithium abundance $\log N(\mathrm{Li})$ and the rotational period $P_{\text {rot }}$.

Results. A set of stellar fundamental parameters is given by our rotating model for each star. We estimate the mass and age to be $1.24 \pm 0.01 M_{\odot}$ and $5.55 \pm 0.20 \mathrm{Gyr}$ for KIC $11395018,1.19 \pm 0.04 M_{\odot}$ and $4.36 \pm 0.29 \mathrm{Gyr}$ for KIC 10273246, and $1.15 \pm 0.03 M_{\odot}$ and $5.68 \pm 0.30 \mathrm{Gyr}$ for KIC 10920273. Moreover, the lithium abundance of our rotating model of the three stars agrees well with the observation.
\end{abstract} \\ Conclusions. The consideration of lithium and the rotational period helped us in obtaining very precise estimates of the star.
}

Key words. stars: evolution - stars: rotation - stars: oscillations

\section{Introduction}

Since the start of the Kepler science operations, several stars have been continuously observed at short-cadence to test and validate the time-series photometry (Gilliland et al. 2010). We studied three of these stars that have clear solar-like oscillation. Their identities in the Kepler Input Catalogue (KIC) are as follows: KIC 11395018, KIC 10273246, and KIC 10920273. They were classified as G4-5IV-V, F9IV-V, and G1-2V, with Kepler magnitudes $(K p)=10.762,10.903$, and 11.926 mag, respectively. Because these stars have been studied in several studies, we refer to them as $\mathrm{C} 1, \mathrm{C} 2$, and $\mathrm{C} 3$ for consistency and convenience. The characteristics of these stars were summarized by Creevey et al. (2012), who provided the fundamental properties of the stars, including the radius, age, luminosity, and rotational rate, and the ranges of mass are $1.34 \pm 0.11$ for $\mathrm{C} 1,1.25 \pm 0.10$ for $\mathrm{C} 2$, and $1.23 \pm 0.11$ for $\mathrm{C} 3$ (see their table 9). Furthermore, 25 individual frequencies of $\mathrm{C} 1$ were provided by Mathur et al. (2011), 30 and 21 individual frequencies of the other stars were derived by Campante et al. (2011).

Creevey et al. (2012) showed that $\mathrm{C} 1$ and $\mathrm{C} 3$ have strong Li absorption lines, which implies a high Li content at the surface, $\log N(\mathrm{Li})=2.6 \pm 0.1$ for $\mathrm{C} 1$ and $\log N(\mathrm{Li})=2.4 \pm 0.1$ for $\mathrm{C} 3$. Considering the empirical $\mathrm{Li}$-age relation established by Sestito et al. (2005), Creevey et al. (2012) then determined that the given $\mathrm{Li}$ abundances would indicate young ages $(0.1-0.4 \mathrm{Gyr}$ for $\mathrm{C} 1$ and 1-3 Gyr for $\mathrm{C} 3$ ), which are incompatible with the asteroseismic ages they determined through the pipeline modeling performed using the average asteroseismic quantities.

Currently, asteroseismology has become not only a useful method for testing theories of stellar structure and evolution, but also a powerful tool for constraining the stellar parameters. Its observational data, such as the large and small frequency separation, provide a very good measurement of the mass, radius, and age of the stars, and the large and small frequency separation are independent of the metallicity in their uncertainty range. In addition, the surface lithium abundance observed in solar-like stars was considered to be an extraordinarily sensitive diagnostic of stellar structure and evolution because lithium is easily burned at relatively low temperature $\left(\sim 2.5 \times 10^{6} \mathrm{~K}\right)$ in the stellar interior. Consequently, the combination of accurate lithium abundance measurement and rotating model helps to provide more precise information about the stellar parameters (do Nascimento et al. 2009; Castro et al. 2011; Li et al. 2012).

In this work, we construct evolutionary models that include microscopic diffusion and mixing driven by rotation. Based on two observed constraints $\left(T_{\text {eff }}\right.$ and $\left.L / L_{\odot}\right)$, two additional observed limits, the lithium abundance $\log N$ (Li) and the rotational period $P_{\text {rot }}$, are considered to constrain the stellar models. For the lithium abundance, we assumed that lithium depletion starts at pre-main-sequence (pre-MS) and experiences dissimilar evolution with different initial rotational rates.

In Sect. 2, we summarize the observations of the three stars. In Sect. 3, the details of the evolutionary models and seismic analysis are presented. In Sect. 4, we describe our modeling results. Finally, the discussion and conclusion are given in Sect. 5.

\section{Observation}

\subsection{Non asteroseismic observational constraints}

The three targets $\mathrm{C} 1, \mathrm{C} 2$, and $\mathrm{C} 3$ were observed by Creevey et al. (2012) using the FIES spectrograph on the Nordic Optical Telescope (NOT) located at the Observatorio del Roque de los 
Table 1. Observational constraint of the three stars.

\begin{tabular}{ccccccc}
\hline \hline Star & $\begin{array}{c}T_{\mathrm{eff}^{a}} \\
(\mathrm{~K})\end{array}$ & $\begin{array}{c}\log g^{a} \\
(\mathrm{dex})\end{array}$ & $\begin{array}{c}{[\mathrm{Fe} / \mathrm{H}]^{a}} \\
(\mathrm{dex})\end{array}$ & $\begin{array}{c}P_{\mathrm{rot}} \\
(\mathrm{days})\end{array}$ & $\begin{array}{c}L^{a} \\
\left(L_{\odot}\right)\end{array}$ & $\begin{array}{c}\log N(\mathrm{Li})^{a} \\
(\mathrm{dex})\end{array}$ \\
\hline $\mathrm{C} 1$ & $5580 \pm 79$ & $3.81 \pm 0.12$ & $+0.19 \pm 0.06$ & $36_{-4.53}^{+6.04 b}$ & $4.2 \pm 1.1$ & $2.6 \pm 0.1$ \\
$\mathrm{C} 2$ & $6050 \pm 100$ & $3.80 \pm 0.11$ & $-0.18 \pm 0.04$ & $23^{c+2.57 d}$ & $5.3 \pm 1.1$ & $<1.9$ \\
$\mathrm{C} 3$ & $5790 \pm 74$ & $4.10 \pm 0.10$ & $-0.04 \pm 0.10$ & $27_{-2.10}^{+6.15 b}$ & $3.6 \pm 1.2$ & $2.4 \pm 0.1$ \\
\hline
\end{tabular}

Notes. ${ }^{(a)}$ Creevey et al. (2012). ${ }^{(b)}$ Mathur et al. (2011). ${ }^{(c)}$ Campante et al. (2011). ${ }^{(d)}$ We estimated the errors according to the PSDs.

Muchachos in La Palma during July and August 2010. Each target was observed twice to give a total exposure time of 46,46 , and $60 \mathrm{~min}$, respectively. This resulted in an signal-to-noise ratio $(\mathrm{S} / \mathrm{N})$ of $\sim 80,90$, and 60 in the wavelength region of 6069-6076 $\AA$. The calibration frames were taken using a Th-Ar lamp. The spectra were reduced using FIESTOOL. The reduced spectra were analyzed by several groups independently using the following methods: SOU (Sousa et al. 2007, 2008), VWA (Bruntt et al. 2010), ROTFIT (Frasca et al. 2006), BIA (Sneden 1973; Biazzo et al. 2011), and NIEM (Niemczura et al. 2005).

The derived atmospheric parameters for the stars for each method are given by Creevey et al. (2012, Table 3 ). We note that Creevey et al. (2012) used the atmospheric parameters from VWA when they constrained the stellar properties. For comparison purposes, we also adopted the effective temperature $T_{\text {eff }}$, the surface gravity $\log \mathrm{g}$, and the metallicity $[\mathrm{Fe} / \mathrm{H}]$ deduced from VWA as the observational constraints. These parameters of the three stars are listed in Table 1.

To determine the stellar rotational period $\left(P_{\text {rot }}\right)$ of these stars, Mathur et al. (2011) and Campante et al. (2011) investigated high $\mathrm{S} / \mathrm{N}$ peaks at the low-frequency end of the power spectral density (PSD). They provided a period of $36_{-4.53}^{+6.04}, 23$, and 27 days for $\mathrm{C} 1, \mathrm{C} 2$, and C3, respectively, where Mathur et al. (2011) used the width of the resolution bin of the PSD of 0.0458 $\mu \mathrm{Hz}$ to compute the error bar for $\mathrm{C} 1$. The errors of $\mathrm{C} 2$ and $\mathrm{C} 3$, which we estimated according to their PSD (Campante et al. $2011)$, are ${ }_{-2.10}^{+2.57}$ days $(0.05 \mu \mathrm{Hz})$ and ${ }_{-4.22}^{+6.15}$ days $(0.08 \mu \mathrm{Hz})$.

\subsection{Asteroseismic constraints}

The Kepler targets C1, C2, and C3 have been observed at short cadence for at least eight months (Q0-4) since the beginning of the Kepler science operations. Observations were briefly interrupted by the planned rolls of the spacecraft and by three unplanned safe-mode events. The duty cycle over these approximately eight months of initial observations was higher than $90 \%$.

The time series has been analyzed by two independent groups using the raw data provided by the Kepler Science Operations Center (Jenkins et al. 2010) and has been subsequently corrected as described by García et al. (2011). The first group used the A2Z pipeline described by Mathur et al. (2010), which investigated the power spectrum of each star to determine $v_{\max }$ and $\langle\Delta v\rangle$ over the range of frequencies from $f_{\min }$ to $f_{\max }$. The second method involves adjusting $\langle\Delta v\rangle$ to make the $\ell=0$ ridge vertical in the Echelle diagram (Huber et al. 2010) over the range of frequencies from $f_{\min }$ to $f_{\max }$.

As previously mentioned, the solar-like oscillations of the three stars have been carefully studied by Mathur et al. (2011, Table 4), and Campante et al. (2011, Tables 5, 6). 25, 30 and 21 individual modes are identified. We adopted the large frequency separation $\langle\Delta v\rangle=47.76 \pm 0.99 \mu \mathrm{Hz},\langle\Delta v\rangle=48.2 \pm 0.5 \mu \mathrm{Hz}$ and $\langle\Delta v\rangle=57.3 \pm 0.8 \mu \mathrm{Hz}$ as a representative value for $\mathrm{C} 1, \mathrm{C} 2$, and
Table 2. Input parameters for the grid calculation.

\begin{tabular}{cccc}
\hline \hline Variable & Minimum & Maximum & $\delta^{a}$ \\
\hline \multicolumn{4}{c}{$\mathrm{C} 1$} \\
$M\left(M_{\odot}\right)$ & 1.23 & 1.45 & 0.02 \\
$Z$ & 0.023 & 0.027 & 0.002 \\
$\alpha$ & 1.75 & 1.95 & 0.20 \\
$V_{\text {ZAMS }}\left(\mathrm{km} \mathrm{s}^{-1}\right)$ & 30 & 50 & 5 \\
\hline \multicolumn{4}{c}{$\mathrm{C} 2$} \\
$M\left(M_{\odot}\right)$ & 1.15 & 1.35 & 0.02 \\
$Z$ & 0.010 & 0.014 & 0.002 \\
$\alpha$ & 1.75 & 1.95 & 0.20 \\
$V_{\text {ZAMS }}\left(\mathrm{km} \mathrm{s}^{-1}\right)$ & 50 & 70 & 5 \\
\hline \multicolumn{4}{c}{$\mathrm{C} 3$} \\
$M\left(M_{\odot}\right)$ & 1.12 & 1.34 & 0.02 \\
$Z$ & 0.014 & 0.018 & 0.002 \\
$\alpha$ & 1.75 & 1.95 & 0.20 \\
$V_{\text {ZAMS }}\left(\mathrm{km} \mathrm{s}^{-1}\right)$ & 40 & 60 & 5 \\
\hline
\end{tabular}

Notes. ${ }^{(a)}$ The value $\delta$ defines the increment between the minimum and maximum parameter values used to construct the models.

C3, respectively. These parameters allowed us to locate the stars in the H-R diagram. The position and their error boxes are useful for theoretical modeling and asteroseismological analysis.

\section{Modeling}

\subsection{Input physics}

We constructed a grid of stellar evolutionary models with the Yale Rotating Stellar Evolution Code (Pinsonneault et al. 1990, 1992; Demarque et al. 2008), which includes diffusion, angular momentum loss, and mixing driven by rotation for different input parameters. These models were computed with the up-to-date OPAL equation-of-state tables EOS2005 (Rogers et al. 2002), the opacities GS98 (Grevesse et al. 1998) supplemented by lowtemperature opacities from Ferguson et al. (2005), the atmosphere following the Eddington $T-\tau$ relation, the NACRE nuclear reaction rates (Angulo et al. 1999), and the mixing length theory (Böhm-Vitense 1958) for convection. The gravitational settling of helium and heavy elements is considered in the stellar model computations using the formulation of Thoul et al. (1994).

Because rotation is considered in our stellar models, the characteristics of a model depend on six parameters: the mass $M$, the age $t$, the mixing-length parameter $\alpha \equiv l / H p$, the rotational period $P_{\text {rot }}$, and two parameters $\left(X_{\text {ini }}, Z_{\text {ini }}\right)$ describing the initial chemical composition of the star. We note that the initial model for each computation was selected on the Hayashi Line because pre-MS lithium-burning is considered. The helium abundance $Y_{\text {ini }}$ of 0.275 was regarded as a constant in all models of the stars. We set the mixing-length parameter $\alpha$ to be 1.75 and 1.95 
for all three stars. In the following, the remaining parameters are given for each star.

For star $\mathrm{C} 1$, the mass range of our grid computation was set to be $1.23-1.45 M_{\odot}$ with a grid size of $0.02 M_{\odot}$. The mass fraction of heavy elements $Z_{\text {ini }}$ was derived from the observed $[\mathrm{Fe} / \mathrm{H}]$ from VWA and $Z_{\odot}$. We used the solar abundance values of Grevesse et al. (1998), viz., $Z_{\odot}=0.0170$ and $(Z / X)_{\odot}=$ 0.0230 . According to the metallicity, the range of $Z_{\text {ini }}$ is from $0.023-0.027$ dex with a grid size of 0.002 dex. The rotational velocity at zero age main sequence $\left(V_{\text {ZAMS }}\right)$ was used to represent the rotational condition of our models. The range of $V_{\mathrm{ZAMS}}$ is $30-50 \mathrm{~km} \mathrm{~s}^{-1}$ with a grid size of $5 \mathrm{~km} \mathrm{~s}^{-1}$. Similarly, we set the ranges of mass, $Z_{\text {ini }}$ and $V_{\text {ZAMs }}$ for the stars $\mathrm{C} 2$ and $\mathrm{C} 3$ with the same grid size as for the star C1. All input parameters of the three stars for the grid calculation are shown in Table 2 .

\subsection{Angular momentum loss}

We adopted the braking law of Kawaler (1988) as the angular momentum loss equation:

$$
\frac{\mathrm{d} J}{\mathrm{~d} t}=\left\{\begin{array}{l}
-K \Omega^{3}\left(R / R_{\odot}\right)^{1 / 2}\left(M / M_{\odot}\right)^{-1 / 2}\left(\Omega \leq \Omega_{\mathrm{sat}}\right) \\
-K \Omega \Omega_{\mathrm{sat}}{ }^{2}\left(R / R_{\odot}\right)^{1 / 2}\left(M / M_{\odot}\right)^{-1 / 2}\left(\Omega>\Omega_{\mathrm{sat}}\right),
\end{array}\right.
$$

where the constant $K$ is correlated with the magnetic field strength, which is commonly taken to be a constant in all stars. $\Omega_{\text {sat }}$ is the angular velocity at which saturation occurs, which is adjustable in the model. Following Bouvier et al. (1997), we set $K=2.0 \times 10^{47} \mathrm{~g} \mathrm{~cm}^{2}$ s and $\Omega_{\text {sat }}=14 \Omega_{\odot}$.

\subsection{Extra-mixing in the radiative region}

Microscopic diffusion and rotation-induced mixing are considered in the radiative region. The transport of angular momentum and element mixing can be described using two diffusion equations as follows (Chaboyer et al. 1995):

$\rho r^{2} \frac{I}{M} \frac{\mathrm{d} \Omega}{\mathrm{d} t}=\frac{\mathrm{d}}{\mathrm{d} r}\left(\rho r^{2} \frac{I}{M} D_{\mathrm{rot}} \frac{\mathrm{d} \Omega}{\mathrm{d} t}\right)$,

$\rho r^{2} \frac{\mathrm{d} X_{i}}{\mathrm{~d} t}=\frac{\mathrm{d}}{\mathrm{d} r}\left[\rho r^{2} D_{\mathrm{m}, 1} X_{i}+\rho r^{2}\left(D_{\mathrm{m}, 2}+f_{\mathrm{c}} D_{\mathrm{rot}}\right) \frac{\mathrm{d} X_{i}}{\mathrm{~d} t}\right]$,

where $\Omega$ is the angular velocity, $X_{i}$ is the mass fraction of chemical species $i$, and $I / M$ is the moment of inertia per unit mass. $D_{\mathrm{m}, 1}$ and $D_{\mathrm{m}, 2}$ are derived from the microscopic diffusion coefficients. $D_{\text {rot }}$ is the diffusion coefficient due to rotation-induced mixing. The details of these three parameters can be found in Chaboyer et al. (1995). The adjustable parameter $f_{\mathrm{c}}$ was used to modify the effects of element mixing caused by rotation. It was determined by requiring that the lithium depletion in the solar model matches the observed depletion (Chaboyer et al. 1995).

\section{Results}

Our study is based on four observed properties $\left(T_{\mathrm{eff}}, L / L_{\odot}\right.$, $\log N(\mathrm{Li})$, and $\left.P_{\text {rot }}\right)$. They were considered to limit the parameters of each of the three stars as constraints. Furthermore, seismic analyses were carried out to match the observed $\langle\Delta v\rangle$ and $\left.v_{n, \ell}\right)$.

\subsection{Stellar models}

To avoid unnecessary repetitions, we take star $\mathrm{C} 1$ as an example.
Firstly, we computed the evolutionary tracks with all possible values of all input parameters. All of the evolutionary tracks in the H-R diagram are plotted in Fig. 1a1 with different line styles and colors. In this step, 280 tracks are found in the ranges of $\alpha$ from 1.75 to 1.95 and $V_{\text {ZAMS }}$ from 30 to $50 \mathrm{~km} \mathrm{~s}^{-1}$. According to Fig. 1a1, we estimate the mass and age of $1.34 \pm 0.11 M_{\odot}$ and $4.50 \pm 1.37 \mathrm{Gyr}$ for $\mathrm{C} 1$. We used the same observational constraints as Creevey et al. (2012), but not all of the tracks fall in the error box. This result is caused by element transport due to the interaction between rotational mixing and diffusion in the radiative region of the stellar interior (Chaboyer et al. 1995; Eggenberger et al. 2010). This type of element transport causes the rotating models to exhibit higher values of effective temperature and luminosity than models without rotation.

Then, the lithium abundance was used as a tracer to constrain the mass and age of the star. Lithium is a key element because it is easily destroyed in the stellar interiors. Its abundance indicates the amount of internal mixing in the stars, and its destruction is strongly mass- and age-dependent (do Nascimento et al. 2009; Li et al. 2012). Since lithium abundance is considered as a constraint, there are 30 tracks that fit the observed lithium abundance $\log N(\mathrm{Li})$. The mixing-length of all these tracks is 1.75 , which can be seen in Fig. 1b1. Because the mixing-length indicates the transfer efficiency of energy and material, the lithium abundance is a function of the mixing-length. Another estimate of mass and age was obtained, which is $1.29 \pm 0.06 M_{\odot}$ and $4.92 \pm 0.87 \mathrm{Gyr}$. Moreover, since the lithium abundance additionally constraints the range of input parameters, the position of the star in the H-R diagram is restricted to a smaller range than in Fig. 1a1.

Furthermore, under the constraint of rotational periods, only seven tracks matched the observed $P_{\text {rot }}$ and are plotted in Fig. $1 \mathrm{c} 1$. The $V_{\mathrm{ZAMS}}$ is also significantly reduced to the range from $40 \mathrm{~km} \mathrm{~s}^{-1}$ to $50 \mathrm{~km} \mathrm{~s}^{-1}$. As shown in Fig. 1c1, the double constraints of lithium abundance and rotational period narrow the ranges of input parameters of the stellar models. The consideration of $P_{\text {rot }}$ helped us estimate mass and age of $\mathrm{C} 1$ even more accurately, they are $1.24 \pm 0.01 M_{\odot}$ and $5.54 \pm 0.21 \mathrm{Gyr}$.

The same method was used for the two other stars C2 and C3; their evolutionary tracks are plotted in Fig. 1a2 to 1c2 for C2 and in Fig. $1 \mathrm{a} 3$ to $1 \mathrm{c} 3$ for C3. Comparing Fig. 1c1, 1c2, and 1c3, we note that star $\mathrm{C} 2$ rotates faster than $\mathrm{C} 1$ and $\mathrm{C} 3$, which is consistent with the results obtained by Li et al. (2012): the faster the star rotates, the faster the lithium dissipates. Moreover, we find that far more tracks for $\mathrm{C} 2$ than $\mathrm{C} 1$ and $\mathrm{C} 3$ in Fig. 1c; this is due to the absence of a lower limit of the lithium abundance for $\mathrm{C} 2$.

Figure 2 plots the evolution of surface abundance of lithium with different initial rotational rates for each star. From Fig. 2, we clearly see that the surface lithium abundance generally decreases with age. When a star evolves in the MS stage, lithium depletion is caused by rotation-induced mixing. We find that rotational effects on lithium-burning in pre-MS stage are quite limited. Fast rotators exhibit almost the same lithium depletion history as slow ones. This is consistent with the results obtained in previous studies, for example, in Mendes et al. (1999). During the MS stage, fast rotators destroy more lithium than slow rotators, because they experience stronger torques and larger shears. For $\mathrm{C} 1$, the model of $1.23 M_{\odot}$ with $V_{\mathrm{ZAMS}}=50 \mathrm{~km} \mathrm{~s}^{-1}$ loses about 0.8 dex lithium during the MS stage, which is 0.3 dex more than the model with $V_{\text {ZAMS }}=30 \mathrm{~km} \mathrm{~s}^{-1}$, as we can see in Fig. 2 a. This trend can be reproduced in Fig. $2 b$ for $\mathrm{C} 2$ and in Fig. 2c for $\mathrm{C} 3$. Hence, the results given by our rotating models imply that 

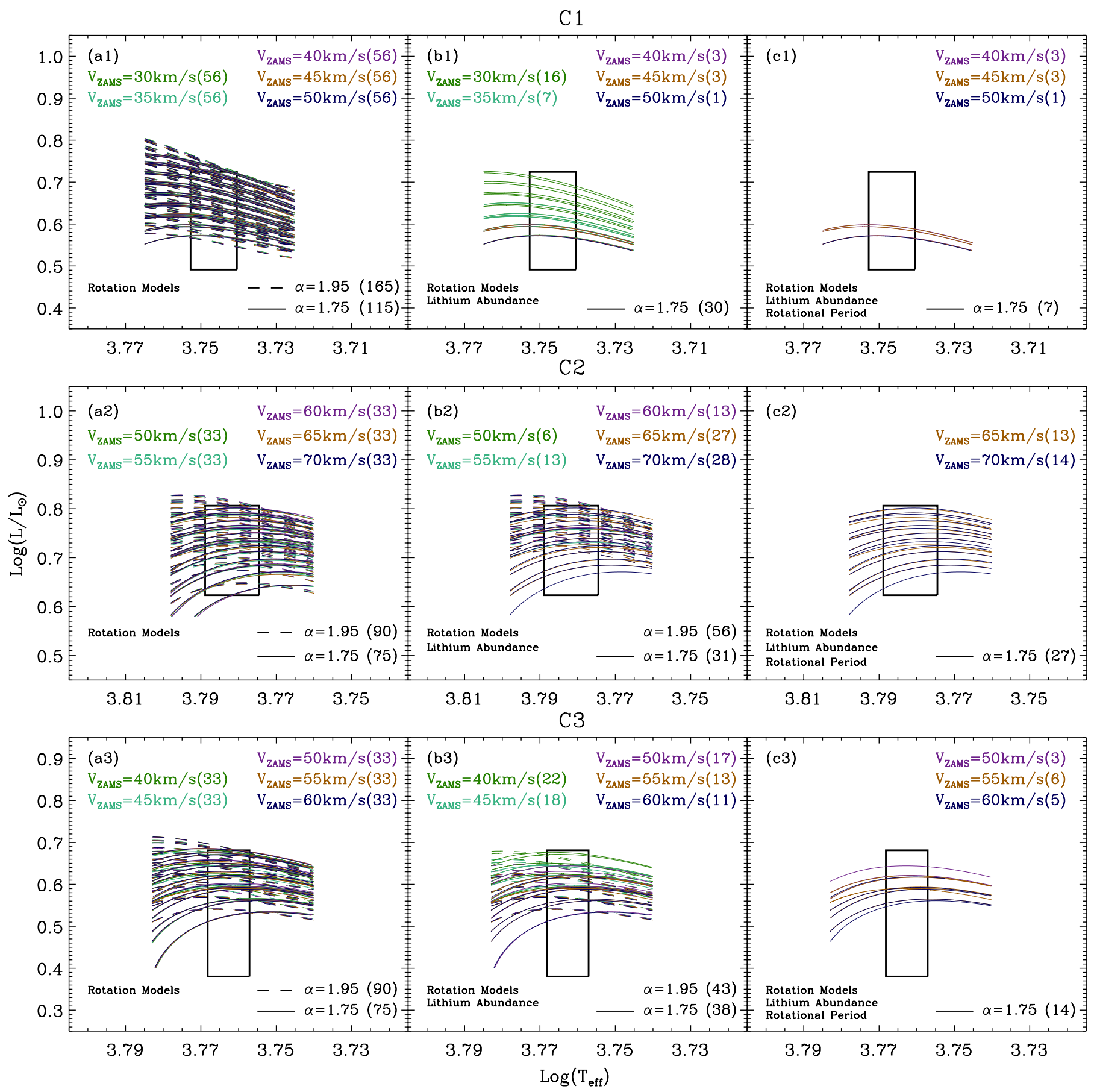

Fig. 1. Evolutionary tracks of the stars in the H-R diagram constrained by different observations. The top panel indicates the stellar models of $\mathrm{C} 1$, the middle panel those of $\mathrm{C} 2$, and the bottom panel those of $\mathrm{C} 3$. The observational constraints are effective temperature and luminosity (left panel), added lithium abundance (middle panel), and added rotational rate (right panel) for each star. The solid line presents $\alpha=1.75$ and the dashed line presents $\alpha=1.95$. The different colors represent rotating models with different initial velocities.

the lithium abundance can be used as a good constraint in stellar modeling.

\subsection{Pulsation analysis}

We used the stellar pulsation code of Guenther (1994) to perform the seismological analysis for the structure models that match all nonasteroseismic constraints. Some analytical and numerical aspects of the code are described in Guenther (1994).

The asteroseismic quantities that we considered are the mean large separation $\langle\Delta v\rangle$, which is defined as the differences between oscillation modes with the same angular degree and consecutive radial order $n$, viz., $\Delta v(n) \equiv v_{n, \ell}-v_{n-1, \ell}$, and the frequencies $v_{n, \ell}$.

To fix the stellar parameters further, we performed an additional comparisons $\chi_{C}^{2}$ between the theoretical and the observed stellar parameters,

$\chi_{C}^{2}=\frac{1}{N} \sum_{i=1}^{N}\left(\frac{C_{i}^{\text {theo }}-C_{i}^{\text {obs }}}{\sigma C_{i}^{\text {obs }}}\right)^{2}$ 
K. Liu et al.: Stellar parameters constrained by lithium abundance and rotation

C1
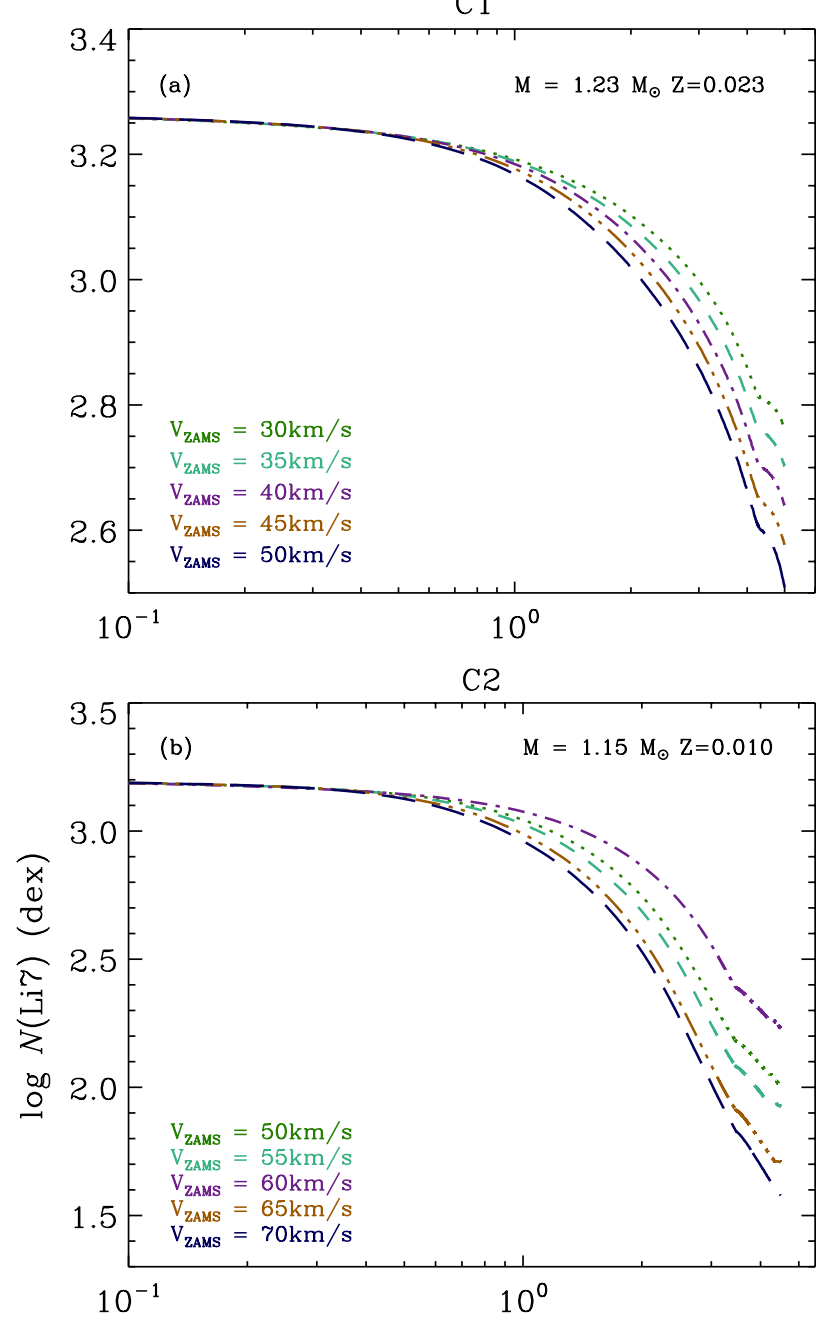

C3

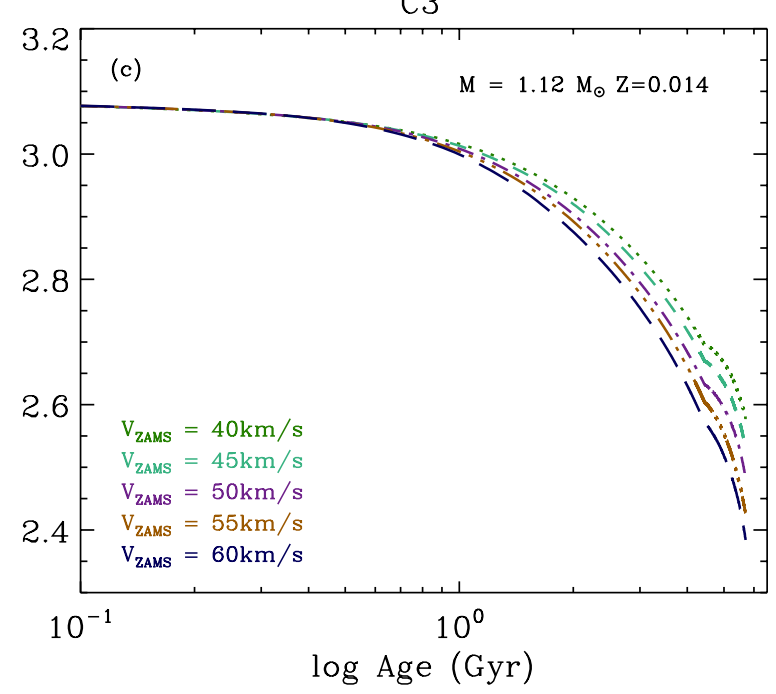

Fig. 2. Lithium depletion with different initial rotational rates. The different lines represent rotating models with different initial velocities.

where $C$ represents the quantities $T_{\text {eff }}, L / L_{\odot}, \log g,[\mathrm{Fe} / \mathrm{H}]$, and the mean large frequency separation $\langle\Delta v\rangle, C_{\text {theo }}$ represents the theoretical values, and $C_{\mathrm{obs}}$ represents the observational values listed in Table 1 . The $\sigma C^{\text {obs }}$ is the errors in these observations, which are also given in Table 1.
C 1
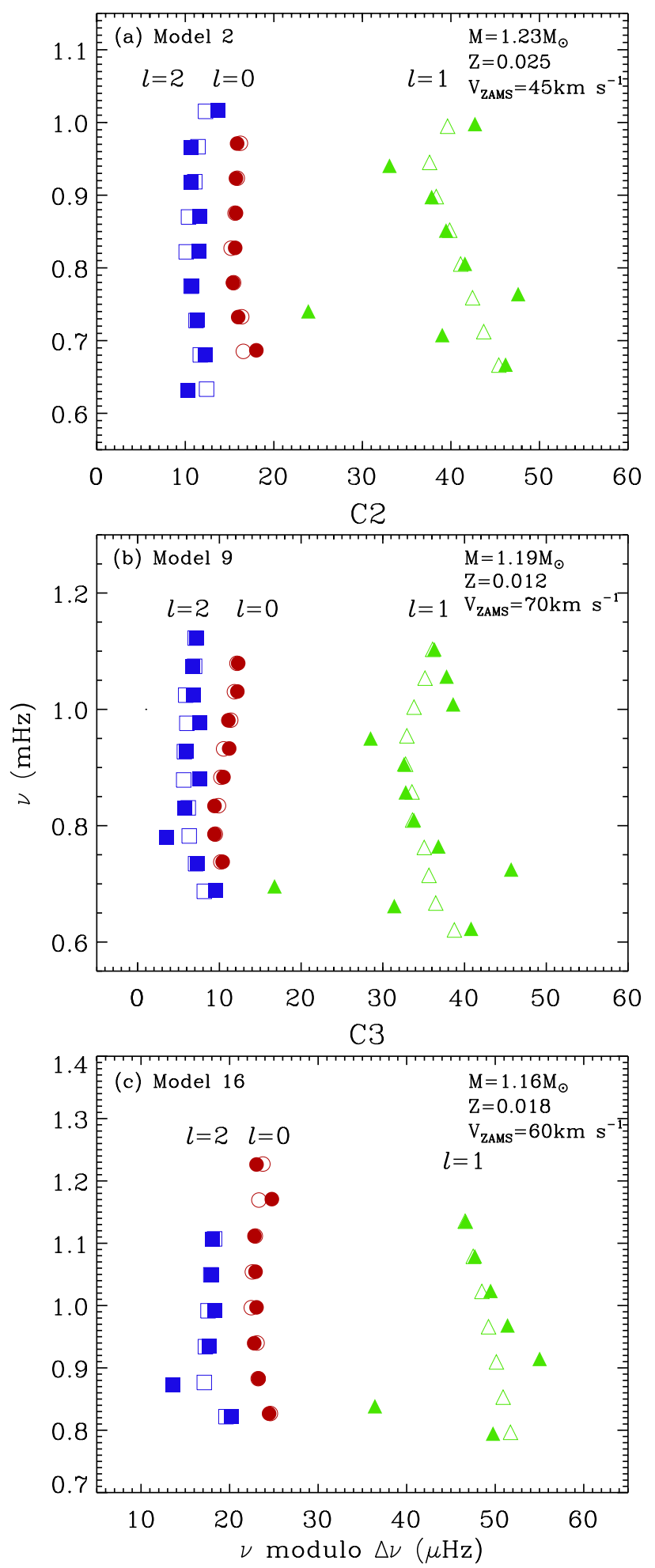

Fig. 3. Échelle diagram corresponding to the mean value of $\langle\Delta v\rangle$ for C1 (Model 2), C2 (Model 9), and C3 (Model 16). The values for modes with $\ell=0, \ell=1$, and $\ell=2$ are shown with circles, triangles, and squares, respectively. The average large frequency separation (47.76 $\mu \mathrm{Hz}$ for $\mathrm{C} 1,48.2 \mu \mathrm{Hz}$ for $\mathrm{C} 2,57.3 \mu \mathrm{Hz}$ for $\mathrm{C} 3$ ) were observed by Mathur et al. (2011) and Campante et al. (2011).

Then, we compared the theoretical frequencies with observed frequencies through the function $\chi_{v}^{2}$,

$\chi_{v}^{2}=\frac{1}{N} \sum_{n, \ell}\left(\frac{v_{n, \ell}^{\text {theo }}-v_{n, \ell}^{\mathrm{obs}}}{\sigma}\right)^{2}$, 
Table 3. Structure models for the seismological analysis.

\begin{tabular}{lcccccccccccc}
\hline \hline Model & $\begin{array}{c}\text { Mass } \\
\left(M_{\odot}\right)\end{array}$ & $Z_{\text {ini }}$ & $\alpha$ & $\begin{array}{c}V_{\text {ZAMs }} \\
\left(\mathrm{km} \mathrm{s}^{-1}\right)\end{array}$ & $\begin{array}{c}\text { Age } \\
(\mathrm{Gyr})\end{array}$ & $\begin{array}{c}T_{\text {eff }} \\
(\mathrm{K})\end{array}$ & $\begin{array}{c}L \\
\left(L_{\odot}\right)\end{array}$ & $\begin{array}{c}R \\
\left(R_{\odot}\right)\end{array}$ & $\begin{array}{c}\log g \\
(\mathrm{dex})\end{array}$ & $\begin{array}{c}P_{\text {rot }} \\
(\text { days })\end{array}$ & $\begin{array}{c}\log N(\mathrm{Li}) \\
(\mathrm{dex})\end{array}$ & $\begin{array}{c}\langle\Delta v\rangle \\
(\mu \mathrm{Hz})\end{array}$ \\
\hline & & & & & & & $\mathrm{C} 1$ & & & & & \\
1 & 1.23 & 0.025 & 1.75 & 40 & 5.51 & 5613 & 3.91 & 2.09 & 3.89 & 41.92 & 2.53 & 47.12 \\
2 & 1.23 & 0.025 & 1.75 & 45 & 5.48 & 5639 & 3.92 & 2.08 & 3.89 & 37.95 & 2.50 & 47.75 \\
3 & 1.23 & 0.027 & 1.75 & 45 & 5.74 & 5550 & 3.71 & 2.09 & 3.89 & 39.79 & 2.50 & 47.42 \\
4 & 1.25 & 0.027 & 1.75 & 45 & 5.35 & 5637 & 3.96 & 2.09 & 3.89 & 38.00 & 2.50 & 47.71 \\
5 & 1.23 & 0.027 & 1.75 & 50 & 5.70 & 5601 & 3.73 & 2.05 & 3.90 & 35.69 & 2.50 & 48.64 \\
\hline & & & & & & $\mathrm{C} 2$ & & & & & & \\
6 & 1.19 & 0.012 & 1.75 & 65 & 4.35 & 6038 & 5.47 & 2.14 & 3.85 & 25.52 & 1.70 & 48.62 \\
7 & 1.23 & 0.014 & 1.75 & 65 & 4.08 & 6030 & 5.61 & 2.17 & 3.85 & 25.50 & 1.75 & 48.31 \\
8 & 1.15 & 0.010 & 1.75 & 70 & 4.64 & 6044 & 5.39 & 2.12 & 3.85 & 25.53 & 1.54 & 48.49 \\
9 & 1.19 & 0.012 & 1.75 & 70 & 4.36 & 6006 & 5.49 & 2.17 & 3.84 & 25.52 & 1.39 & 47.78 \\
10 & 1.21 & 0.012 & 1.75 & 70 & 4.07 & 6075 & 5.81 & 2.18 & 3.84 & 25.56 & 1.67 & 47.75 \\
11 & 1.23 & 0.014 & 1.75 & 70 & 4.08 & 6026 & 5.61 & 2.18 & 3.85 & 25.59 & 1.71 & 48.23 \\
\hline & & & & & & $\mathrm{C} 3$ & & & & & & \\
12 & 1.16 & 0.016 & 1.75 & 50 & 5.49 & 5828 & 4.17 & 2.01 & 3.90 & 33.14 & 2.40 & 57.09 \\
13 & 1.18 & 0.018 & 1.75 & 50 & 5.39 & 5828 & 4.12 & 1.99 & 3.91 & 33.18 & 2.44 & 57.99 \\
14 & 1.12 & 0.014 & 1.75 & 55 & 5.98 & 5764 & 3.88 & 1.98 & 3.89 & 32.78 & 2.30 & 57.25 \\
15 & 1.16 & 0.018 & 1.75 & 55 & 5.81 & 5738 & 3.89 & 2.00 & 3.90 & 33.20 & 2.43 & 57.41 \\
16 & 1.16 & 0.018 & 1.75 & 60 & 5.83 & 5717 & 3.88 & 2.01 & 3.90 & 31.33 & 2.36 & 56.95 \\
17 & 1.18 & 0.018 & 1.75 & 60 & 5.38 & 5827 & 4.13 & 2.00 & 3.91 & 27.49 & 2.30 & 57.88 \\
\hline
\end{tabular}

where $N$ is the total number of modes, $v_{n, \ell}^{\text {theo }}$ and $v_{n, \ell}^{\text {obs }}$ are the theoretical and the observed frequencies for each spherical degree $\ell$ and the radial order $n$, and $\sigma$ represents the uncertainty of the observed frequencies (Mathur et al. 2010; Campante et al. 2011).

Combining $\chi_{C}^{2}(<1)$ with $\chi_{v(\ell=0,2)}^{2}(<20)$, we found 17 models, viz., Model 1-5 for C1, Model 6-11 for C2, and Model 12-17 for $\mathrm{C} 3$, which probably are the best-fitting models. The details of all of the selected models of the three stars are shown in Table 3. The properties of the 17 models provide the final estimates of the stellar parameters of the three stars. The pulsation analysis provides even better estimates of the mass and age, which are $1.24 \pm 0.01 M_{\odot}$ and $5.55 \pm 0.20$ Gyr for $\mathrm{C} 1,1.19 \pm 0.04 M_{\odot}$ and $4.36 \pm 0.29 \mathrm{Gyr}$ for $\mathrm{C} 2$, and $1.15 \pm 0.03 M_{\odot}$ and $5.68 \pm 0.30 \mathrm{Gyr}$ for $\mathrm{C} 3$.

Among these models, we chose the one with the lowest value of $\chi_{v}^{2}$ for each star, viz., Model 2, Model 9, and Model 16. We compare the observational and theoretical frequencies of the three models in Table 4. In addition, their échelle diagrams are plotted in Fig. 3. We note that the mixed characteristics of the mode with $\ell=1$ are fuzzy for the theoretical frequencies of all three stars. This may be because the interior structure of the star has been changed by rotation-induced mixing of elements, for instance, the size of the inner core and the stellar mean density. Hence, we might need to consider some other physical processes in detail in our future work.

\section{Discussion and conclusions}

Based on general observed features, we used two additional observed quantities, the lithium abundance and the rotational period, to better estimate the stellar parameters and address the problem of the incompatibility between the lithium abundance and the age in stars $\mathrm{C} 1$ and $\mathrm{C} 3$. Therefore, theoretical analyses of these two additional features can significantly constrain the ranges of the input parameters and ensure a better self-consistent stellar model.

To make our method clear and easy to compare with Creevey et al. (2012), according to the results in Fig. 1. we summarized the stellar parameters of the three stars determined by different constraints in Table 5. Previous results are listed in line 1 for each star, lines 2-5 show the stellar parameters derived in each step of our method. First, we estimated the mass and age of the stars using only the classical features $T_{\text {eff }}$ and $L / L_{\odot}$. The uncertainty of the results is approximately $0.08 M_{\odot}$ and $1.10 \mathrm{Gyr}$. Additionally, we note that our results are not identical to those obtained by Creevey et al. (2012). The differences are caused by element transport due to the interaction between rotational mixing and diffusion in the radiative region of the stellar interior, which causes rotating models to have a higher $T_{\text {eff }}$ and luminosity. Because we added the lithium abundance and rotational period to our analysis, more accurate determinations were obtained. The lithium abundance helped us to improve the uncertainty of mass and age to $0.06 M_{\odot}$ and 0.90 Gyr. Next, based on the above results, we used the rotational period to constrain stellar parameters. Very precise estimates were obtained in this step, specifically, $\Delta M \sim 0.04 M_{\odot}$ and $\Delta \tau \sim 0.50$ Gyr. Finally, the pulsation analysis was performed.

After this analysis, we gave the final estimates for mass and age of the three stars, which are $1.24 \pm 0.01 M_{\odot}$ and $5.55 \pm 0.20 \mathrm{Gyr}$ for $\mathrm{C} 1,1.19 \pm 0.04 M_{\odot}$ and $4.36 \pm 0.29 \mathrm{Gyr}$ for $\mathrm{C} 2$, and $1.15 \pm 0.03 M_{\odot}$ and $5.68 \pm 0.30 \mathrm{Gyr}$ for $\mathrm{C} 3$, respectively. The results locate the stars more precisely in the H-R diagram. This result is reasonable because lithium depletes as a function of mass, metallicity, rotational rates and age, while the rotational period decreases with age during the main sequence. Therefore, these two additional features can significantly constrain the input parameter ranges and improve the self-consistency and accuracy of the stellar model.

In addition, the change of the initial velocity causes different degrees of rotation-mixing in the radiative region, resulting in different processes of lithium depletion. Thus, the rotating model can be used to explain the incompatibility between lithium abundance and age in stars $\mathrm{C} 1$ and $\mathrm{C} 3$. We conclude that the lithium depletion, rotating evolution, and pulsation analysis are important to place constraints on the stellar fundamental parameters. work. 
K. Liu et al.: Stellar parameters constrained by lithium abundance and rotation

Table 4. Comparison of observed and theoretical frequencies for C1 (Model 2), C2 (Model 9), and C3 (Model 16).

\begin{tabular}{|c|c|c|c|c|c|c|}
\hline \multicolumn{3}{|c|}{$\mathrm{C} 1$} & \multicolumn{2}{|c|}{$\mathrm{C} 2$} & \multicolumn{2}{|c|}{$\mathrm{C} 3$} \\
\hline$\ell$ & $\begin{array}{c}\text { Observational } \\
\text { frequencies }(\mu \mathrm{Hz})\end{array}$ & $\begin{array}{r}\text { Theoretical } \\
\text { frequencies }(\mu \mathrm{Hz})\end{array}$ & $\begin{array}{c}\text { Observational } \\
\text { frequencies }(\mu \mathrm{Hz})\end{array}$ & $\begin{array}{r}\text { Theoretical } \\
\text { frequencies }(\mu \mathrm{Hz})\end{array}$ & $\begin{array}{c}\text { Observational } \\
\text { frequencies }(\mu \mathrm{Hz})\end{array}$ & $\begin{array}{r}\text { Theoretical } \\
\text { frequencies }(\mu \mathrm{Hz})\end{array}$ \\
\hline 0 & $686.66 \pm 0.32$ & 685.19 & $737.90 \pm 0.30$ & 737.68 & $826.66 \pm 0.25^{a}$ & 826.81 \\
\hline 0 & $732.37 \pm 0.18$ & 732.77 & $785.40 \pm 0.20$ & 785.50 & $882.77 \pm 0.20$ & 882.73 \\
\hline 0 & $779.54 \pm 0.14$ & 779.61 & $833.90 \pm 0.20$ & 834.39 & $939.58 \pm 0.16$ & 939.90 \\
\hline 0 & $827.55 \pm 0.15$ & 827.09 & $883.50 \pm 0.20$ & 883.19 & $997.14 \pm 0.18$ & 996.55 \\
\hline 0 & $875.40 \pm 0.16$ & 875.30 & $932.70 \pm 0.50$ & 932.02 & $1054.33 \pm 0.30$ & 1053.97 \\
\hline 0 & $923.16 \pm 0.19$ & 923.31 & $981.10 \pm 0.30$ & 981.39 & $1111.51 \pm 0.25$ & 1111.61 \\
\hline 0 & $971.05 \pm 0.28$ & 971.42 & $1030.70 \pm 0.40$ & 1030.34 & $1170.77 \pm 0.33^{a}$ & 1169.31 \\
\hline 0 & $\ldots$ & $\ldots$ & $1079.30 \pm 0.20$ & 1079.15 & $1226.34 \pm 0.33^{a}$ & 1227.06 \\
\hline 1 & $667.05 \pm 0.22^{a}$ & 666.28 & $622.80 \pm 0.20$ & 620.75 & $794.65 \pm 0.32^{b}$ & 796.62 \\
\hline 1 & $707.66 \pm 0.19$ & 712.34 & $661.90 \pm 0.50$ & 666.97 & $838.61 \pm 0.25^{b}$ & 853.09 \\
\hline 1 & $740.29 \pm 0.17^{b}$ & - & $695.75 \pm 0.27^{b}$ & - & $914.52 \pm 0.16$ & 909.63 \\
\hline 1 & $763.99 \pm 0.18$ & 758.84 & $724.70 \pm 0.20$ & 714.64 & $968.19 \pm 0.13$ & 966.04 \\
\hline 1 & $805.74 \pm 0.13$ & 805.25 & $764.30 \pm 0.30$ & 762.56 & $1023.58 \pm 0.14$ & 1022.59 \\
\hline 1 & $851.37 \pm 0.11$ & 851.78 & $809.80 \pm 0.20$ & 809.63 & $1079.10 \pm 0.31$ & 1078.91 \\
\hline 1 & $897.50 \pm 0.15$ & 898.02 & $857.30 \pm 0.20$ & 858.06 & $1135.36 \pm 0.31^{c}$ & 1135.30 \\
\hline 1 & $940.50 \pm 0.15$ & 945.03 & $905.60 \pm 0.30$ & 905.78 & $\ldots$ & ... \\
\hline 1 & $997.91 \pm 0.33$ & 994.82 & $950.00 \pm 0.30$ & 954.44 & $\ldots$ & $\ldots$ \\
\hline 1 & $\ldots$ & $\ldots$ & $1008.60 \pm 0.40$ & 1003.81 & $\ldots$ & $\ldots$ \\
\hline 1 & $\ldots$ & $\ldots$ & $1056.30 \pm 0.20$ & 1053.66 & $\ldots$ & $\ldots$ \\
\hline 1 & ... & ... & $1103.30 \pm 0.40$ & 1103.09 & $\ldots$ & ... \\
\hline 2 & $631.19 \pm 1.36^{a}$ & 633.28 & $688.50 \pm 0.70$ & 687.15 & $822.39 \pm 0.28^{a}$ & 821.79 \\
\hline 2 & $680.88 \pm 0.45$ & 680.33 & $734.80 \pm 0.60$ & 734.59 & $873.10 \pm 0.32^{a, d}$ & 876.64 \\
\hline 2 & $727.78 \pm 0.30$ & 727.61 & $779.50 \pm 0.40$ & 782.31 & $934.49 \pm 0.22$ & 934.07 \\
\hline 2 & $774.92 \pm 0.16$ & 774.81 & $830.30 \pm 0.40$ & 830.69 & $992.44 \pm 0.13$ & 991.66 \\
\hline 2 & $823.50 \pm 0.16$ & 822.02 & $880.60 \pm 0.50$ & 878.64 & $1049.36 \pm 0.39$ & 1049.28 \\
\hline 2 & $871.29 \pm 0.21$ & 870.04 & $927.50 \pm 0.40$ & 927.22 & $1106.76 \pm 0.34$ & 1107.00 \\
\hline 2 & $918.10 \pm 0.28$ & 918.51 & $977.60 \pm 0.40$ & 976.02 & $\ldots$ & $\ldots$ \\
\hline 2 & $965.83 \pm 0.23$ & 966.63 & $1025.30 \pm 1.30$ & 1024.38 & $\ldots$ & $\ldots$ \\
\hline 2 & $1016.61 \pm 0.73^{e, f}$ & 1015.24 & $1073.70 \pm 0.20$ & 1073.97 & $\ldots$ & $\ldots$ \\
\hline 2 & $\ldots$ & $\ldots$ & $1122.70 \pm 0.40^{a, d}$ & 1122.54 & $\ldots$ & $\ldots$ \\
\hline
\end{tabular}

Notes. ${ }^{(a)}$ Frequencies not among the minimal set. ${ }^{(b)} \ell=1$ mixed mode. ${ }^{(c)}$ Mode close to the second harmonic of the inverse of the long-cadence period. ${ }^{(d)}$ Possible $\ell=2$ mixed mode introduced a posteriori. ${ }^{(e)}$ Uncertain identification. ${ }^{(f)}$ Mode-tagging changed a posteriori from $\ell=0$ to $\ell=2$.

Table 5. Stellar parameters of the three stars determined by different methods and previous studies.

\begin{tabular}{|c|c|c|c|c|c|c|}
\hline Star & Observational constraints & $\begin{array}{c}M \\
\left(M_{\odot}\right) \\
\end{array}$ & $\begin{array}{c}\tau \\
(\mathrm{Gyr}) \\
\end{array}$ & $\begin{array}{c}R \\
\left(R_{\odot}\right) \\
\end{array}$ & $\begin{array}{l}T_{\text {eff }} \\
(\mathrm{K})\end{array}$ & $\begin{array}{c}L \\
\left(L_{\odot}\right) \\
\end{array}$ \\
\hline \multirow[t]{5}{*}{$\mathrm{C} 1$} & Previous results & $1.34 \pm 0.11$ & $4.5 \pm 0.5$ & $2.22 \pm 0.05$ & 5547 & $4.2 \pm 1.1$ \\
\hline & Classical features $^{(*)}$ & $1.34 \pm 0.11$ & $4.50 \pm 1.37$ & $2.27 \pm 0.27$ & $5580 \pm 79$ & $4.41 \pm 0.89$ \\
\hline & Classical features $+\log N(\mathrm{Li})$ & $1.29 \pm 0.06$ & $4.92 \pm 0.87$ & $2.20 \pm 0.19$ & $5580 \pm 79$ & $4.44 \pm 0.75$ \\
\hline & Classical features $+\log N(\mathrm{Li})+P_{\text {rot }}$ & $1.24 \pm 0.01$ & $5.54 \pm 0.21$ & $2.06 \pm 0.05$ & $5604 \pm 54$ & $3.84 \pm 0.13$ \\
\hline & Classical features $+\log N(\mathrm{Li})+P_{\text {rot }}+\langle\Delta v\rangle+v_{n, \ell}$ & $1.24 \pm 0.01$ & $5.55 \pm 0.20$ & $2.07 \pm 0.02$ & $5594 \pm 45$ & $3.84 \pm 0.13$ \\
\hline \multirow[t]{5}{*}{$\mathrm{C} 2$} & Previous results & $1.25 \pm 0.10$ & $3.7 \pm 0.6$ & $2.11 \pm 0.05$ & 6047 & $5.3 \pm 1.1$ \\
\hline & Classical features $^{(*)}$ & $1.22 \pm 0.07$ & $4.45 \pm 0.98$ & $2.10 \pm 0.27$ & $6050 \pm 100$ & $5.30 \pm 1.10$ \\
\hline & Classical features $+\log N(\mathrm{Li})$ & $1.22 \pm 0.07$ & $4.24 \pm 0.77$ & $2.13 \pm 0.24$ & $6050 \pm 100$ & $5.49 \pm 0.91$ \\
\hline & Classical features $+\log N(\mathrm{Li})+P_{\text {rot }}$ & $1.21 \pm 0.06$ & $4.29 \pm 0.72$ & $2.12 \pm 0.21$ & $6050 \pm 100$ & $5.45 \pm 0.88$ \\
\hline & Classical features $+\log N(\mathrm{Li})+P_{\text {rot }}+\langle\Delta v\rangle+v_{n, \ell}$ & $1.19 \pm 0.04$ & $4.36 \pm 0.29$ & $2.15 \pm 0.03$ & $6041 \pm 35$ & $5.60 \pm 0.21$ \\
\hline \multirow[t]{5}{*}{$\mathrm{C} 3$} & Previous results & $1.23 \pm 0.11$ & $5.0 \pm 1.9$ & $1.90 \pm 0.06$ & 5789 & $3.6 \pm 1.2$ \\
\hline & Classical features $^{a}$ & $1.18 \pm 0.06$ & $5.68 \pm 1.11$ & $1.99 \pm 0.24$ & $5790 \pm 74$ & $4.02 \pm 0.78$ \\
\hline & Classical features $+\log N(\mathrm{Li})$ & $1.17 \pm 0.05$ & $5.77 \pm 1.02$ & $2.01 \pm 0.20$ & $5790 \pm 74$ & $4.07 \pm 0.68$ \\
\hline & Classical features $+\log N(\mathrm{Li})+P_{\text {rot }}$ & $1.16 \pm 0.04$ & $5.66 \pm 0.62$ & $1.96 \pm 0.12$ & $5795 \pm 69$ & $4.00 \pm 0.41$ \\
\hline & Classical features $+\log N(\mathrm{Li})+P_{\mathrm{rot}}+\langle\Delta v\rangle+v_{n, \ell}$ & $1.15 \pm 0.03$ & $5.68 \pm 0.30$ & $1.99 \pm 0.02$ & $5772 \pm 56$ & $4.02 \pm 0.15$ \\
\hline
\end{tabular}

Notes. ${ }^{(a)}$ The classical features include $T_{\text {eff }}$ and $L / L_{\odot}$. The differences in results between Creevey et al. (2012) and us were a result of the rotating model. 
Acknowledgements. We are grateful to the Kepler Science Team for their constructive suggestions and valuable remarks to improve the manuscript. Funding for this Discovery mission is provided by NASA's Science Mission Directorate. This work is supported by grants 10933002 and 11273007 from the National Natural Science Foundation of China, and the Fundamental Research Funds for the Central Universities.

\section{References}

Angulo, C., Arnould, M., Rayet, M., et al. 1999, Nucl. Phys. A, 656, 3 Biazzo, K., Randich, S., \& Palla, F. 2011, A\&A, 525, A35

Böhm-Vitense, E. 1958, ZAp, 46, 108

Bouvier, J., Forestini, M., \& Allain, S. 1997, A\&A, 326, 1023

Bruntt, H., Bedding, T. R., Quirion, P. O., et al. 2010, MNRAS, 405, 1907

Campante, T. L., Handberg, R., Mathur, S., et al. 2011, A\&A, 534, A6

Castro, M., do Nascimento, J. D., Jr., Biazzo, K., et al. 2011, A\&A, 526, A17

Chaboyer, B., Demarque, P., Guenther, D. B., et al. 1995, ApJ, 446, 435

Creevey, O. L., Doğan, G., Frasca, A., et al. 2012, A\&A, 537, A111

Demarque, P., Guenther, D. B., Li, L. H., Mazumdar, A., \& Straka, C. W. 2008, Ap\&SS, 316, 31

do Nascimento, J. D., Jr., Castro, M., Meléndez, J., et. al. 2009, A\&A, 501, 687

Eggenberger, P., Meynet, G., Maeder, A., et al. 2010, A\&A, 519, A116

Ferguson, J. W., Alexander, D. R., Allard, F., et al. 2005, ApJ, 623, 585
Frasca, A., Guillout, P., Marilli, E., et al. 2006, A\&A, 454, 301

García, R. A., Hekker, S., Stello, D., et al. 2011, MNRAS, 414, L6

Gilliland, R. L., Jenkins, J. M., Borucki, W. J., et al. 2010, ApJ, 713, 160

Grevesse, N., \& Sauval, A. J. 1998, Space Sci. Rev., 85, 161

Guenther, D. B. 1994, ApJ, 422, 400

Huber, D., Matthews, J. M., Croll, B., et al. 2010, ApJ, 723, 1607

Jenkins, J. M., Caldwell, D. A., Chandrasekaran, H., et al. 2010, ApJ, 713, L87

Kawaler, S. D. 1988, ApJ, 333, 236

Li, T. D., Bi, S. L., Chen, Y. Q., et al. 2012, ApJ, 746, 143

Mathur, S., García, R. A., Régulo, C., et al. 2010, A\&A, 511, A46

Mathur, S., Handberg, R., Campante, T. L., et al. 2011, ApJ, 733, 95

Mendes, L. T. S., D’Antona, F., \& Mazzitelli, I. 1999, A\&A, 341, 174

Niemczura, E., \& Połubek, G. 2005, Mem. Soc. Astron. It., 8, 210

Pinsonneault, M. H., Kawaler, S. D., \& Demarque, P. 1990, ApJS, 74, 501

Pinsonneault, M. H., Deliyannis, C. P., \& Demarque, P. 1992, ApJS, 78, 179

Rogers, F. J., \& Nayfonov, A. 2002, ApJ, 576, 1064

Sestito, P., \& Randich, S. 2005, A\&A, 442, 615

Sneden, C. 1973, ApJ, 184, 839

Sousa, S. G., Santos, N. C., Israelian, G., Mayor, M., \& Monteiro, M. J. P. F. G. 2007, A\&A, 469, 783

Sousa, S. G., Santos, N. C., Mayor, M., et al. 2008, A\&A, 487, 373

Thoul, A. A., Bahcall, J. N., \& Loeb, A. 1994, ApJ, 421, 828 